\title{
A prospective cohort study of the accuracy and safety of robot-assisted minimally invasive spinal surgery
}

Mingxing Fan ${ }^{1,2 \dagger}$, Yanming Fang ${ }^{1,2 \dagger}$, Qi Zhang ${ }^{1,2}$, Jingwei Zhao ${ }^{1,2}$, Bo Liu ${ }^{1,2}$ and Wei Tian ${ }^{1,2^{*}}$

\begin{abstract}
Background: Robot-assisted open surgery (RA-OS) is now commonly used in traditional open-exposure spinal screw placement surgery. With the help of robots, robot-assisted minimally invasive surgery (RA-MIS) can achieve less bleeding and less tissue damage in percutaneous screw insertion. While the research comparing the safety and accuracy of screw placement between RA-MIS and RA-OS is insufficient. This study aims to compare the effects of RA-MIS and RA-OS in thoracic and lumbar spine.

Methods: This was a prospective cohort study evaluating 208 patients undergoing robot-assisted screw insertions from July 2020 to September 2021. Age, BMI, gender, screws accuracy, screws Gertzbein-Robbins grade, small joint invasion and perioperative outcomes (operation time, blood loss, postoperative hospital stay, comorbidity) were collected. A subgroup analysis was also performed according to disease, namely fracture, spondylolisthesis, and disc herniation. Data were analyzed using Stata/MP 14.0. Wilcoxon's signed rank test, Kruskal-Wallis test and Fisher's exact test were used for statistical tests and $p<0.05$ was considered statistically significant.
\end{abstract}

Results: A total of 1030 screws were inserted; 368 minimally invasive screws and 662 open screws. The acceptability of screw insertion in the RA-MIS and RA-OS was $97.3 \%$ and $95.6 \%$ respectively. There was no statistical difference between the RA-MIS group and RA-OS group in age $(p=0.106)$, gender $(p=0.074)$, BMI $(p=0.181)$ and comorbidity $(p=0.203)$. Compared with RA-OS, RA-MIS had less blood loss $(p<0.001)$ and shorter postoperative hospital stay $(p=0.008)$. In the minimally invasive surgery group, the fracture subgroup had smaller screw deviation, less blood loss, and shorter operation time compared with the other subgroups $(p<0.01)$. Specifically, RA-MIS significantly reduced the postoperative hospital stay of patients with spondylolisthesis compared with RA-OS $(p<0.01)$.

Conclusion: RA-OS and RA-MIS had equal accuracy and safety. Compared with open surgery, minimally invasive surgery reduced blood loss in each subgroup and shortened the postoperative hospital stay in the spondylolisthesis subgroup. Compared with the other subgroups under minimally invasive surgery, the fracture subgroup had less blood loss and shorter operation time.

Clinical trial registration: NCT04040868. Registered 1 March 2019, https://clinicaltrials.gov/ct2/show/NCT04040868? cond=Accuracy+Study+of+Robot-assisted+Screw+Insertion+in+Spinal+Surgery\&draw=2\&rank=1.

*Correspondence: tianweijst_2021@126.com

${ }^{+}$Mingxing Fan and Yanming Fang contributed equally to this article

${ }^{1}$ Spine Department, Beijing Jishuitan Hospital, Beijing, China

Full list of author information is available at the end of the article

\section{Background}

Spine surrounds the spinal cord and nerves, playing important roles in support, protection, and movement. Spinal diseases can cause spinal cord and nerve damage that seriously affect patients' health and quality of life $[10$, 
18]. Pedicle screw internal fixation technology is one of the most important methods to restore the spine to its pre-injury structure and function. In traditional open spinal surgery, some screws have large deviations from the expected path owing to the narrow intraoperative view, complex anatomical structures, and insufficient surgeons' proficiency. Screw placement accuracy in open surgery is low and ranges from 69 to $94 \%[6,16]$. Fortunately, the emergence of orthopedic robots has solved this problem. Compared with traditional spine surgery, orthopedic robot-assisted surgery has higher screw placement safety, with an accuracy of between 94.5 and $99 \%[8,11,12]$, which has also reduced the possibility of nerve damage secondary to inaccurate pedicle screw placement.

Traditional open surgery requires stripping of a large amount of paravertebral muscle and soft tissue, which may lead to several postoperative complications. Additionally, tissue damage and adhesion may cause postoperatively back stiffness and muscle weakness. With the assistance of robots, doctors can perform a minimally invasive surgical method of percutaneous pedicle screw fixation called minimally invasive surgery. It has the advantages of less soft tissue damage, less intraoperative bleeding, shorter hospital stay, and improved quality of life. $[8,14,20,21]$. However, comparison studies of the effects of RA-MIS vs RA-OS are preliminary, and evaluations of these approaches for different spinal diseases is lacking. There is still no definite answer to whether robot-assisted surgery can be used in different spinal diseases safely and effectively due to different pathophysiological mechanisms.

In this study, we prospectively collected data of 208 patients with thoracic or lumbar spinal disease in our Department of Spine Surgery from July 2020 to September 2021. Patients were divided into RA-MIS and RA-OS groups. Differences in the accuracy, safety, and perioperative outcomes were evaluated according to disease subgroups. This article laid the foundation for studying the clinical effects of RA-MIS and RA-OS in different diseases.

\section{Methods}

\section{Study design and patient selection}

We prospectively enrolled patients in Spine Surgery Department from July 2020 to September 2021 and divided patients into RA-MIS and RA-OS groups according to disease condition and patients' willing. The sample size was estimated using a non-inferiority test of two independent samples. The test level was set at 0.025 , test power was 0.2 , and the loss to follow-up ratio was 0 . According to a ratio of 1:1.5, 81 patients in the RA-MIS group and 121 patients in the RA-OS were needed. The final total number of enrolled patients was 208 patients, including 79 in the RA-MIS group and 129 in the RA-OS group, with a total of 1030 screws inserted.

The inclusion criteria were as follows: patients aged 18-85 years undergoing robot-assisted pedicle screw internal fixation owing to thoracic or lumbar diseases. The exclusion criteria were as follows: patients with previous spinal surgery; history of tumors or tuberculosis; multiple traumas; concurrent disease, such as severe hypertension and heart disease; and patients not suitable to undergo robotic screw placement. The patients were further divided into fracture, spondylolisthesis, and disc herniation subgroups according to the diagnosis at admission. We defined the fracture subgroup as patients with pain symptoms, malformations, abnormal activities, or bone friction feeling, with imaging examinations showing that the integrity and continuity of the spine was interrupted. We defined the spondylolisthesis subgroup as patients with symptoms related to spondylolisthesis, with imaging studies indicating that a vertebral body had slipped position relative to its adjacent vertebral bodies. We defined the disc herniation subgroup as patients presenting with symptoms related to a herniated vertebral disc, with imaging examinations suggesting that the disc was herniated. This study was approved by the Ethics Committee and patients' agreement.

\section{Robotic system and surgical procedure}

Robot-assisted surgery was performed using the TiRobot orthopedic robot system (TINAVI Medical Technologies, Beijing, China). Orthopedic surgery robot system is composed of host, mechanical arm, surgery planning and control software, optical tracking system, main control trolley and navigation and positioning toolkit. Its operation involves image and optical data acquisition, image registration, surgical planning, mechanical positioning and other steps. The robot uses intraoperative images for surgical planning, and uses robotic arm for movement and holding to achieve precise positioning of implants. Its working process mainly includes 4 steps: (1) Surgical planning, the doctor uses the supporting surgical planning and control software to design the surgical path on the intraoperative images, and select the appropriate implant; (2) Spatial calibration, obtain the spatial coordinates of the surgical path through a certain positioning algorithm and device; (3) Path positioning, control the robot to automatically move according to the spatial coordinates of the surgical path, and move the surgical tool to the target surgical path; (4) Auxiliary surgery, the doctor performs surgical operations under the guidance of a robotic arm (Fig. 1).

The patient was placed in the prone position on the $\mathrm{X}$-ray/operating table (Fig. 2). After inducing general anesthesia, a $\mathrm{C}$-arm X-ray machine (ARCADIS Orbic 
A
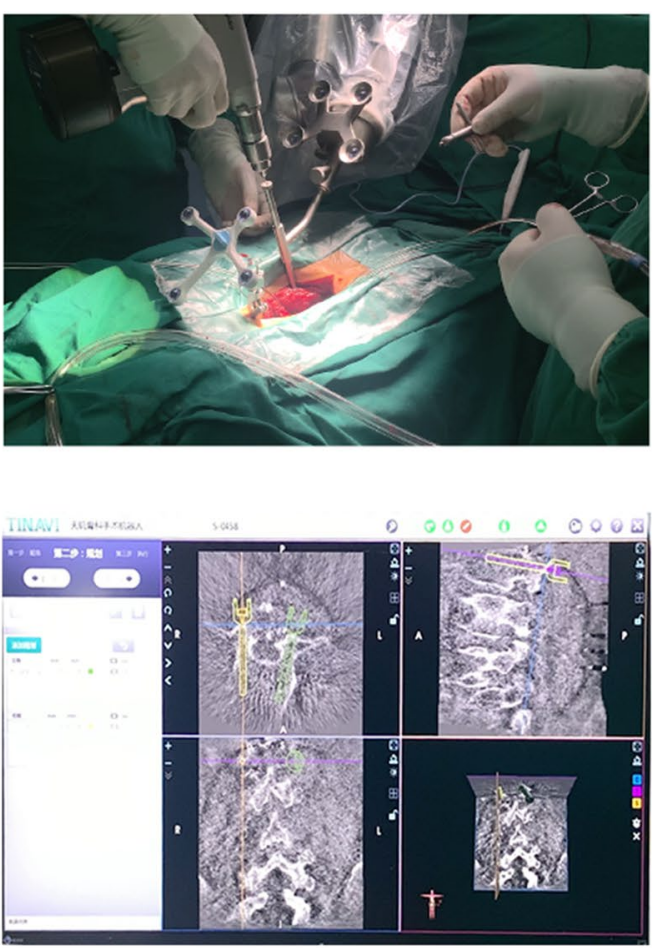

$\mathrm{B}$

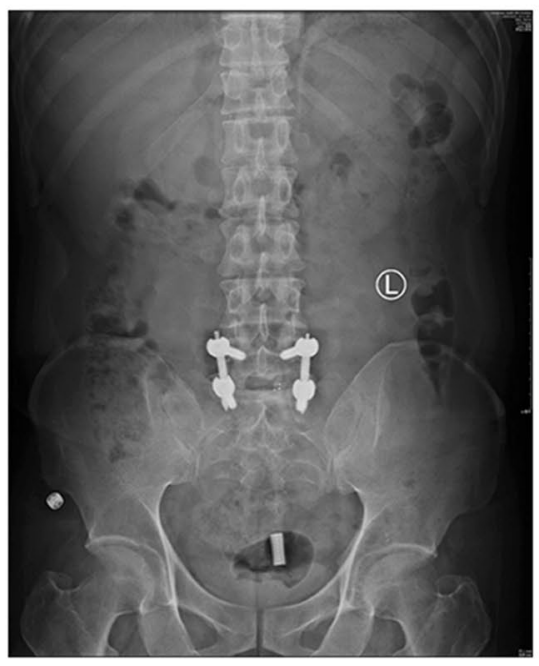

$\mathrm{b}$

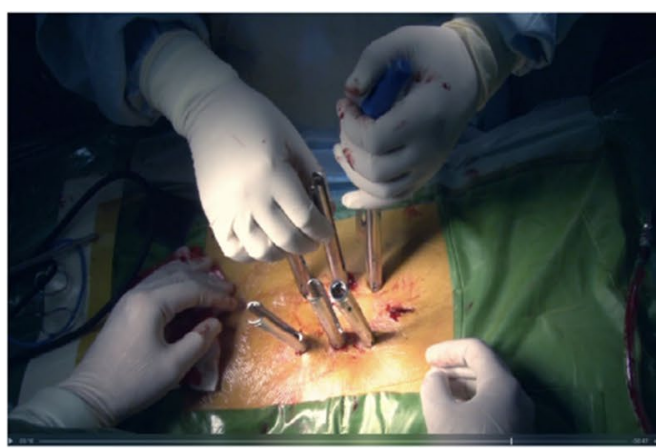

a
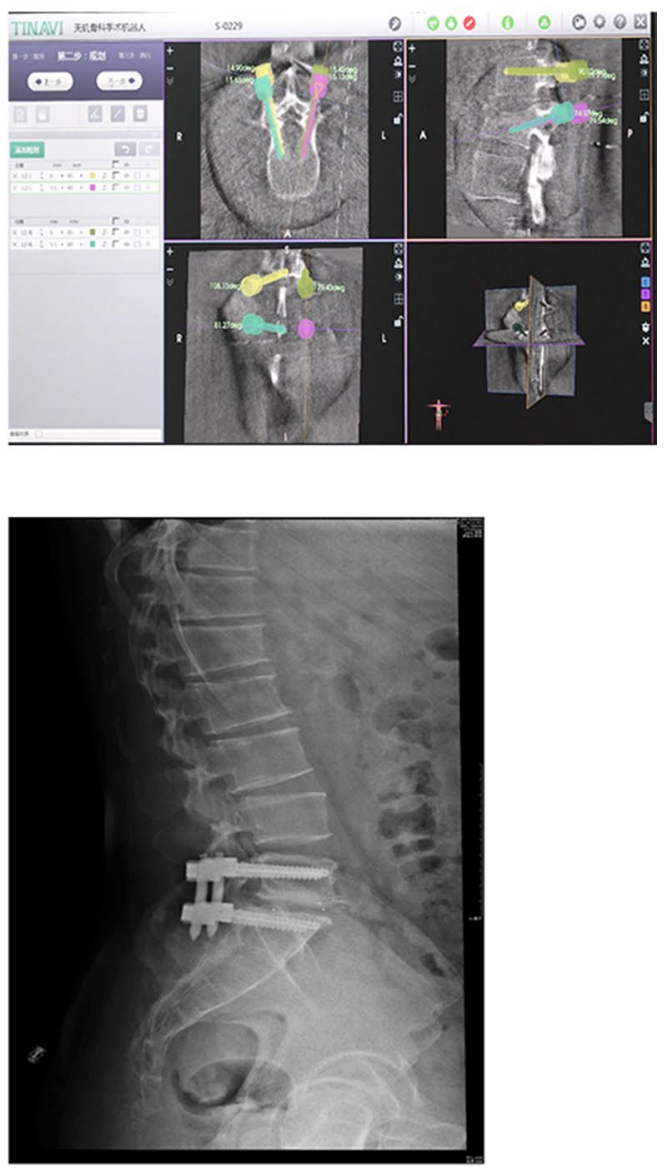

Fig. 1 Clinical photographs, intraprocedural imaging and radiographs. A Surgery photo of RA-OS; a surgery photo of RA-MIS; B: Screw insertion design of cervical spine; $\mathbf{b}$ Screw insertion design of lumbar spine; $\mathbf{C}$ and $\mathbf{c}$ radiographs after lumbar surgery

3D C-arm; Siemens, Erlangen, Germany) was used to locate the operating area. RA-OS exposed the surgical field according to the requirements of traditional surgery. (RA-MIS firstly exposed and fixed the patient tracer, then exposed the surgical field under robot guidance.) The tracer was usually fixed on the spinous process of the upper vertebral body adjacent to the surgical segment. After image acquisition using intraoperative real-time three-dimensional navigation, surgeons planned and designed the surgery using the robot control software and selected the spine level, screw diameter, length, direction and angle. After the guide was installed, the robotarm advanced to the planned position, and a sleeve was placed on the guide to monitor the positioning accuracy in real time. A powered system was used to drill into the K-wire along the sleeve. Hollow screws can be inserted 


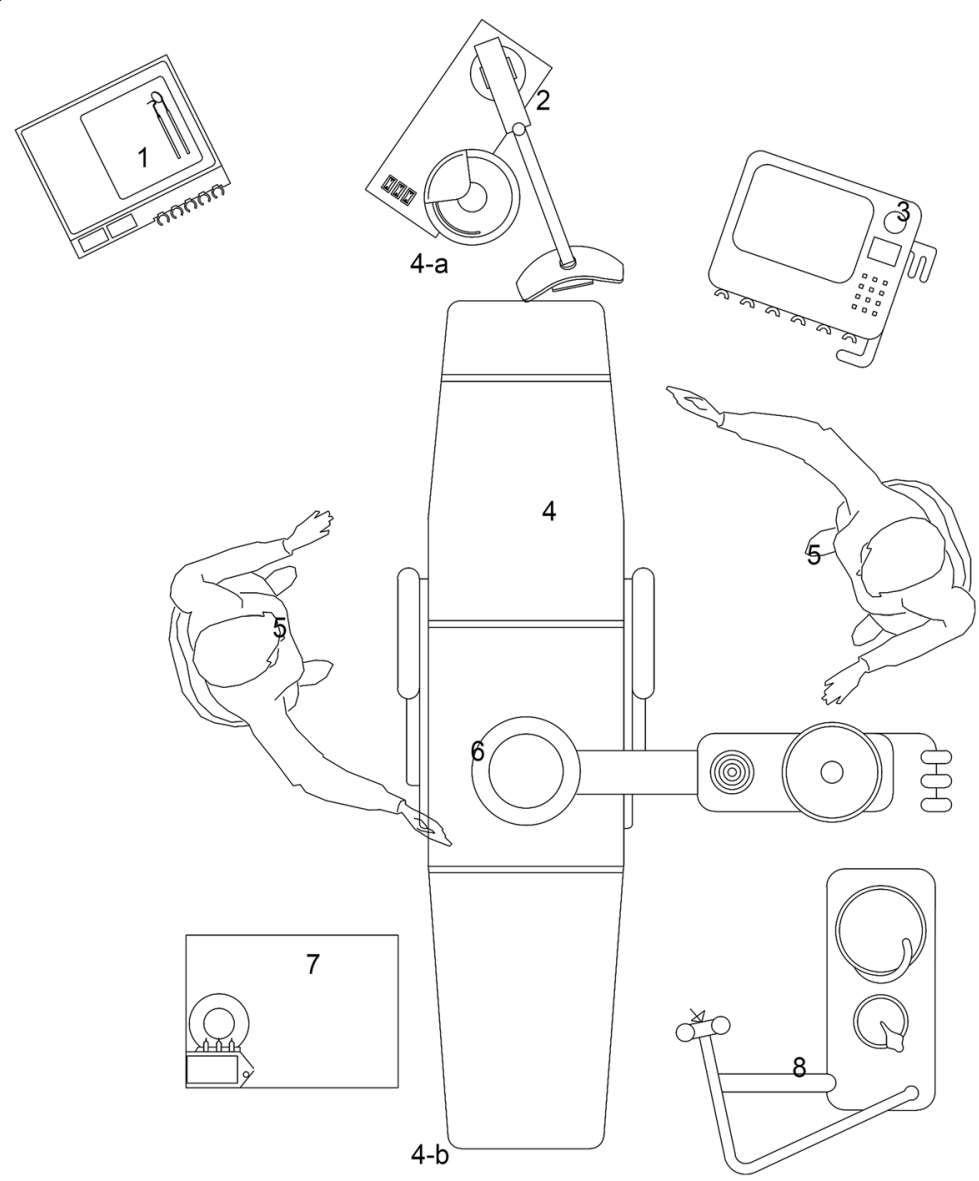

Fig. 2 Schematic diagram of the placement of the patient and the robotic equipment

Legend

1. Anesthetics and other monitoring equipment

2. Optical tracking camera

3. Robot controller

4. Operating table

4-a. Head side 4-b. Feet side

5. Surgeon

6. C-arm X-ray

7. Instrument table

8. Robot directly along the K-wire; with ordinary screws, a hollow drill was used to prepare the screw channel before insertion. After screw insertion, C-arm X-ray fluoroscopy was used to assess screw placement, and bilateral connecting rods were installed followed by suturing the incision (Fig. 3).

\section{Outcome measures}

This study analyzed the patients' basic demographic characteristics, namely sex, age, body mass index (BMI), and perioperative outcomes, namely operative time, intraoperative blood loss, postoperative hospital stay, and comorbidity. According to postoperative computed tomography $(\mathrm{CT})$ findings, the number of screw facet joint invasions, screw deviation (distance that the screw deviated from the designed trajectory), and screw safety were assessed. Gertzbein and Robbins scale [7] was used for estimating screw safety as follows: Grade A: the screw is completely in the pedicle; Grade B: the distance of the screw breaking through the pedicle cortex is $<2 \mathrm{~mm}$; Grade C: the distance of the screw breaking through the

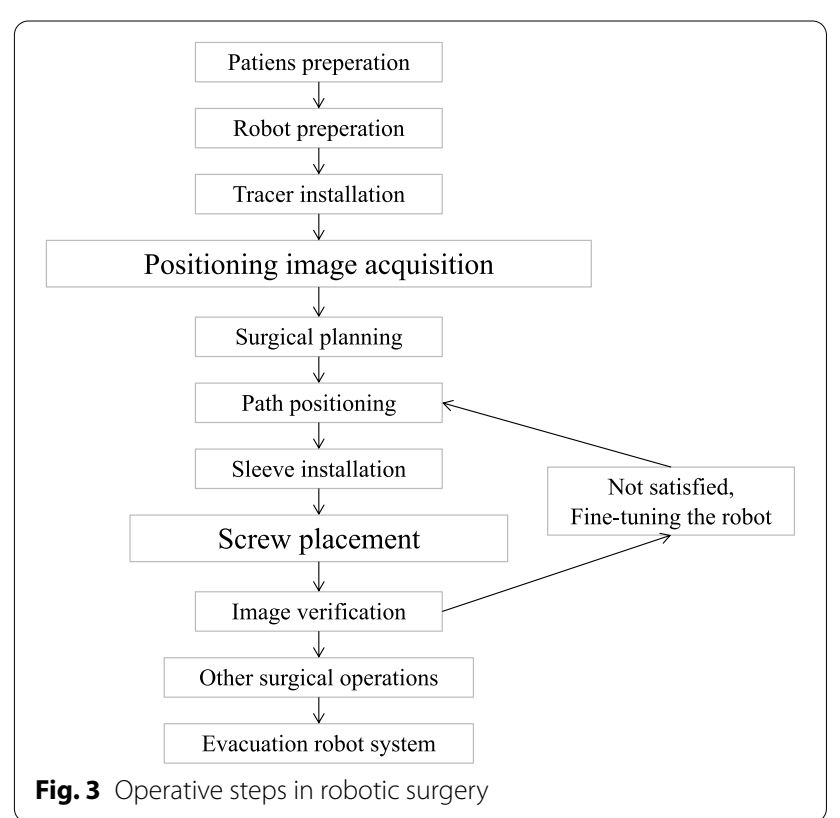


pedicle cortex is $\geq 2 \mathrm{~mm}$ and $<4 \mathrm{~mm}$; Grade $\mathrm{D}$ : the distance of the screw breaking through the pedicle cortex is $\geq 4 \mathrm{~mm}$ and $<6 \mathrm{~mm}$; and Grade $\mathrm{E}$ : the distance of the screw breaking through the pedicle cortex is $>6 \mathrm{~mm}$. We calculated the acceptability rate of screw placement using the following formula: [(number of grade A screws + number of grade B screws) / total number of screws $\times 100 \%]$.

\section{Statistical analysis}

Data were analyzed using Stata/MP 14.0 (College Station, TX, USA). The Shapiro-Wilk's test was used for normally-distributed data, with analysis of variance (ANOVA). Measurement data conforming to normal distribution and homogeneity of variance was expressed as mean \pm standard deviation. Measurement data that not conforming to normal distribution or homogeneity of variance was expressed as median (75\% quartile-25\% quartile). Wilcoxon's signed rank test was performed for comparisons between two groups, and the Kruskal-Wallis test was performed for comparisons between multiple subgroups. Numerical data are expressed as frequency (percent). Comparisons between groups were also performed using Fisher's exact test or the chi-square test, and the rank mean difference (RMD) was calculated. $p<0.05$ was considered statistically significant.

\section{Results}

\section{Demographic data}

Two hundred and eight patients were enrolled in this study with 79 in the RA-MIS group and 129 in the RA-OS group. A total of 1030 screws were inserted. There was no statistical difference between the RA-MIS group and RA-OS group for age [56 years (64-50) vs 60 years (64$54), p=0.106]$, gender, BMI $\left[25.69 \mathrm{~kg} / \mathrm{m}^{2}(27.68-23.53)\right.$ vs $25 \mathrm{~kg} / \mathrm{m}^{2}$ (27.63-22.58), $\left.p=0.181\right]$ and comorbidity [1 vs $0, p=0.0 .203)$ ] (Table 1 ).

\section{Perioperative outcomes and Screw characteristics in generally}

There was no statistical difference in operation time between the two groups. Blood loss in the RA-MIS group was less than that in the RA-OS group [100 mL (200-50) vs $200 \mathrm{~mL}(400-200), p<0.001]$ and the postoperative hospital stay [5 days $(6-4)$ vs 5 days $(7-4), p=0.008$ ] was shorter (Table 1). One patient developed postoperative superficial incision infection in the RA-MIS group, and was cured through local wound disinfection and short-term antibiotic treatment. There was no statistical difference between the two groups on comorbidities $(p=0.203)$. No others critical issues were identified else.

A total of 1030 screws were inserted: 368 screws in the RA-MIS group, namely 50 in the thoracic spine

Table 1 Demographic data, perioperative outcomes and screw placement results for the RA-MIS and RA-OS

\begin{tabular}{|c|c|c|c|c|}
\hline & $\begin{array}{l}\text { Minimally invasive surgery } \\
(\mathrm{N}=79)\end{array}$ & Open surgery $(\mathrm{N}=129)$ & Statistics & $p$ Value \\
\hline Age (year) & $56(64-50)$ & $60(64-54)$ & $Z=-1.615$ & 0.106 \\
\hline Gender & & & $x^{2}=3.2020$ & 0.074 \\
\hline Male & $40(44.6 \%)$ & $49(44.4 \%)$ & & \\
\hline Female & $39(55.4 \%)$ & $80(55.6 \%)$ & & \\
\hline $\mathrm{BMI}\left(\mathrm{kg} / \mathrm{m}^{2}\right)$ & $25.69(27.68-23.53)$ & $25(27.63-22.58)$ & $Z=1.1139$ & 0.181 \\
\hline Operation time (minutes) & $150(180-100)$ & $135(180-120)$ & $Z=-0.034$ & 0.973 \\
\hline Intraoperative blood loss (mL) & $100(200-50)$ & $200(400-200)$ & $Z=-6.347$ & $<0.001$ \\
\hline Postoperative hospital stay (days) & $5(6-4)$ & $5(7-4)$ & $Z=-2.658$ & 0.008 \\
\hline Comorbidity & 1 & 0 & $x^{2}=1.6203$ & 0.203 \\
\hline Number of screws & 368 & 662 & $x^{2}=0.0000$ & 1.000 \\
\hline Thoracic spine & $50(13.6 \%)$ & $90(13.6 \%)$ & & \\
\hline Lumbosacral Spine & $318(86.4 \%)$ & $572(86.4 \%)$ & & \\
\hline Small joint invasion & $9(2.4 \%)$ & $20(3.0 \%)$ & Fisher & 0.697 \\
\hline GRS grade & & & Fisher & 0.470 \\
\hline Grade A & 331 (90.0\%) & $583(88.1 \%)$ & & \\
\hline Grade B & $27(7.3 \%)$ & $50(7.5 \%)$ & & \\
\hline Grade $(A+B)$ & $358(97.3 \%)$ & $633(95.6 \%)$ & & \\
\hline Grade C & $9(2.4 \%)$ & $25(3.8 \%)$ & & \\
\hline Grade D & $1(0.3 \%)$ & $4(0.6 \%)$ & & \\
\hline Screw deviation (mm) & $1.37(2.05-0.86)$ & $1.34(2.21-0.90)$ & $Z=-1.048$ & 0.2948 \\
\hline
\end{tabular}

Age, BMI, operation time, blood loss, postoperative hospital stay and screw deviation were expressed as median ( $75 \%$ quartile-25\% quartile). Gender, comorbidity, number of screws, facet joint invasion and Gertzbein and Robbins (GRS) grade are expressed as frequency (percentage). $B M I$ body mass index 
and 318 in the lumbosacral spine. Among these, there were 331 grade A screws (90.0\%) and 27 grade B screws $(7.3 \%)$. In the RA-OS group, 662 pedicle screws were inserted with 90 in the thoracic spine and 572 in the lumbosacral spine. Among these screws, 583 (88.1\%) were grade A screws, and 50 (7.5\%) were grade B screws. The acceptability rate of screw placement was $97.3 \%$ in the RA-MIS and $95.6 \%$ in the RA-OS (Table 1). There was no statistical difference between the two groups regarding screw distribution according to the spinal levels $\left(x^{2}=0.000 ; p=1.000\right)$, number of small joint invasions (Fisher's, $p=0.697$ ), safety classification (Fisher's, $p=0.470$ ) and screw deviation $(\mathrm{Z}=-1.048 ; p=0.295)$.

\section{Screw characteristics in the subgroups}

Screw deviation was statistically different in the subgroup of RA-MISs (Table 2). Screw deviation in fracture subgroup was smaller than that in spondylolisthesis subgroup $[1.11 \mathrm{~mm} \quad(1.75-0.69)$ vs $1.33 \mathrm{~mm}(2.09-0.97), \mathrm{RMD}=37.73, p<0.003]$ and disc herniation subgroup [1.11 $\mathrm{mm}(1.75-0.69)$ vs $1.54 \mathrm{~mm}$ (2.15-1.03), $\mathrm{RMD}=53.04, p<0.001]$. A similar trend was observed in the RA-OS group. Screw accuracy in fracture patients was significantly higher than in the spondylolisthesis patients $[1.01 \mathrm{~mm}(1.42-0.68)$ vs $1.64 \mathrm{~mm}(2.42-1.06), \mathrm{RMD}=132.10, p<0.001]$ and the disc herniation patients $[1.01 \mathrm{~mm}(1.42-0.68) \mathrm{vs}$ $1.47 \mathrm{~mm}(2.42-0.96), \mathrm{RMD}=113.74, p<0.001]$. Additionally, the precision of RA-MIS was higher than for RA-OS [1.33 mm (2.09-0.97) vs $1.64 \mathrm{~mm}(2.42-1.06)$; $p=0.036]$ in the spondylolisthesis subgroup (Fig. 4). In the fracture subgroup or disc herniation subgroup, the choice of MIS or RA-OS had no significant statistically impact on screw deviation.

\section{Perioperative outcomes in the subgroups}

There were statistical differences in intraoperative blood loss and operation time between the subgroups undergoing RA-MIS. Intraoperative blood loss was lower in the fracture subgroup than in the spondylolisthesis subgroup [ $50 \mathrm{~mL}$ (90-50) vs $100 \mathrm{~mL}(200-50), \mathrm{RMD}=16.57$; $p=0.004)]$ and disc herniation subgroup [50 $\mathrm{mL}(90-50)$ vs $200 \mathrm{~mL}(200-100), \mathrm{RMD}=25.55 ; p<0.001]$. Similarly, operation time in the fracture subgroup was shorter than in the spondylolisthesis subgroup [100 $\min (135-90)$ vs $150 \mathrm{~min}(180-150), \mathrm{RMD}=20.22 ; p<0.001]$ and disc herniation subgroup [100 min (135-90) vs 150 min (225$120), \mathrm{RMD}=17.30 ; p=0.003]$. There were also statistical differences in intraoperative blood loss and postoperative hospital stay in the different subgroups in the RA-OS group. Intraoperative blood loss in the fracture subgroup was less than in the spondylolisthesis subgroup [100 mL $(200-50)$ vs $200 \mathrm{~mL}(400-200), \mathrm{RMD}=37.41 ; p<0.001]$ and the disc herniation subgroup [100 $\mathrm{mL}(200-50)$ vs $200 \mathrm{~mL}(400-200), \mathrm{RMD}=36.44 ; p<0.001]$. Postoperative hospital stay in the spondylolisthesis subgroup was longer than that in the fracture subgroup [120 min (170$112.5)$ vs $135 \mathrm{~min}(180-120), \mathrm{RMD}=29.00, p=0.001]$ and disc herniation subgroup [120 $\min (170-112.5)$ vs $150 \min (180-120), \mathrm{RMD}=20.10 ; p=0.003]$. Compared with RA-OS, RA-MIS was associated with less intraoperative blood loss in each subgroup $(p<0.001)$ and shorter postoperative hospital stay in the spondylolisthesis subgroup $(\mathrm{Z}=-3.491 ; p<0.001)$ (Table 3$)$.

\section{Discussion}

Internal screw fixation is an important method to treat spinal injuries and instability. With freehand screw insertion, screw is easier to deviate from the designed trajectory owing to the narrow visual field, and may enter the spinal canal and damage the spinal cord or the nerve

Table 2 Screw placement in the disease subgroups

\begin{tabular}{|c|c|c|c|c|c|c|}
\hline & & Fracture & Spondylolisthesis & Disc herniation & Statistics & $p$ Value \\
\hline \multirow[t]{2}{*}{ Number of patients } & Minimally Invasive & 24 & 28 & 27 & $x^{2}=4.5278$ & 0.104 \\
\hline & Open & 24 & 46 & 59 & & \\
\hline \multirow[t]{2}{*}{ Number of screws } & Minimally Invasive & 122 & 120 & 126 & $x^{2}=10.9273$ & 0.006 \\
\hline & Open & 160 & 222 & 280 & & \\
\hline \multirow[t]{2}{*}{ Grade $(A+B) /$ Total } & Minimally Invasive & $121 / 122(99.18 \%)$ & $115 / 120(95.8 \%)$ & $122 / 126(96.83 \%)$ & & \\
\hline & Open & $157 / 160(98.13 \%)$ & $213 / 222$ (95.95\%) & $269 / 280(96.07 \%)$ & & \\
\hline \multirow[t]{2}{*}{ Screw deviation (mm) } & Minimally Invasive & $1.11(1.75-0.69) * \Delta$ & $1.33(2.09-0.97)$ & $1.54(2.15-1.03)$ & $x^{2}=16.240$ & $<0.001$ \\
\hline & Open & $1.01(1.42-0.68) * \Delta$ & $1.64(2.42-1.06)$ & $1.47(2.42-0.96)$ & $x^{2}=50.400$ & $<0.001$ \\
\hline
\end{tabular}

Screw deviation was expressed as median (75\% quartile-25\% quartile), and the number of screws and patients were expressed in frequency. $R M D$ rank means difference

* Represents statistical significance compared with the spondylolisthesis group $(p<0.05)$, and $\Delta$ represents statistical significance compared with disc herniation group $(p<0.05)$ undergoing same surgical method (minimally invasive group or open group) 


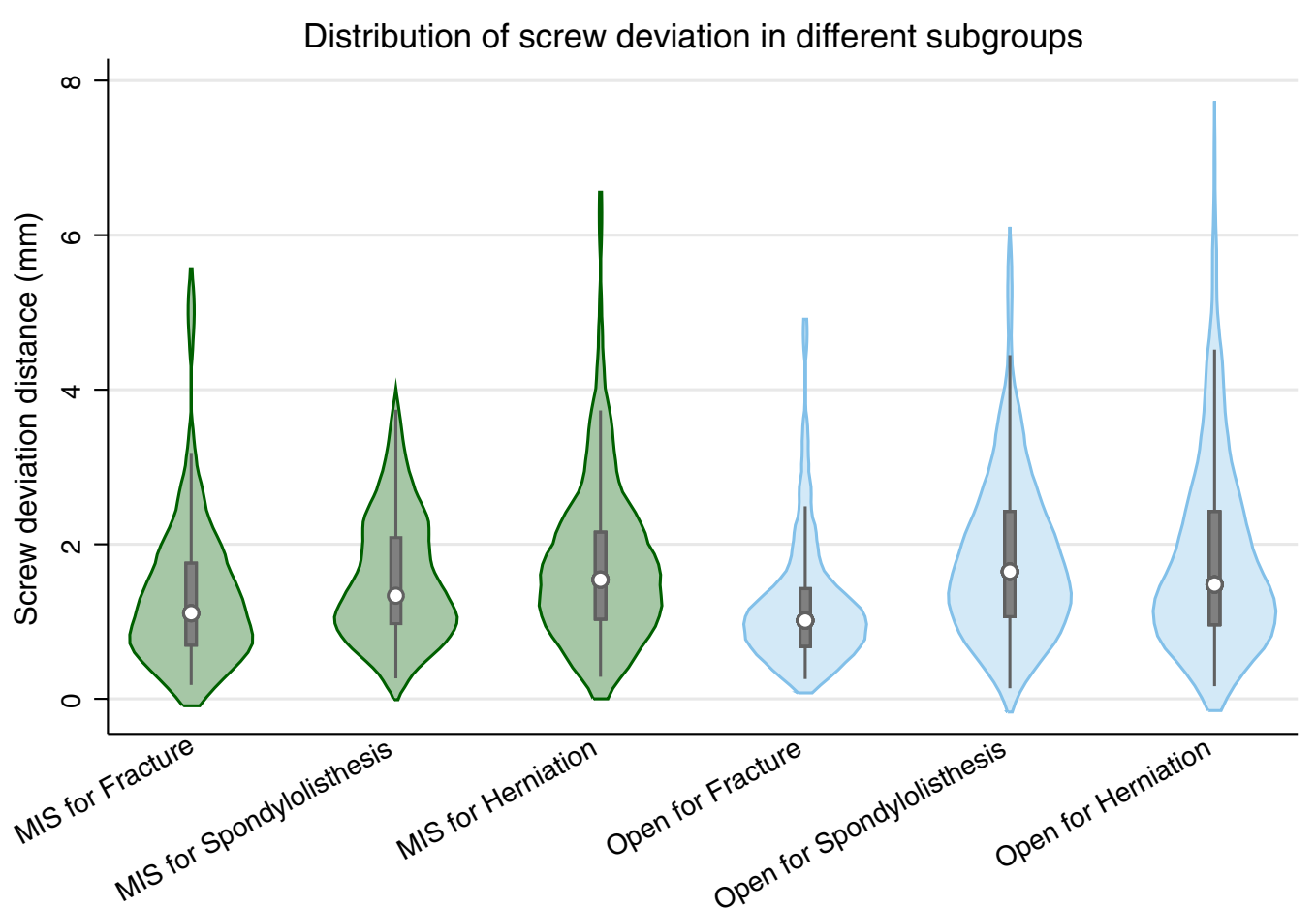

Fig. 4 Violin plots of the screw deviation distance distributions. The ordinate is the deviation distance, the width of the violin is the relative frequency distribution, and the center of the violin is the box plot. MIS minimally invasive surgery

Table 3 Perioperative outcomes in the disease subgroups

\begin{tabular}{lllllll}
\hline & & Fracture & Spondylolisthesis & Disc herniation & Statistics & $\boldsymbol{p}$ Value \\
\hline Intraoperative blood loss $(\mathrm{mL})$ & Minimally Invasive & $50(90-50){ }^{*} \Delta$ & $100(200-50)$ & $200(200-100)$ & $x^{2}=17.665$ & $<0.001$ \\
& Open & $100(200-50){ }^{*} \Delta$ & $200(400-200)$ & $200(400-200)$ & $x^{2}=21.381$ & $<0.001$ \\
Operation time (minutes) & Minimally Invasive & $100(135-90){ }^{*} \Delta$ & $150(180-150)$ & $150(225-120)$ & $x^{2}=12.831$ & 0.002 \\
& Open & $120(170-112.5)$ & $135(180-120)$ & $150(180-120)$ & $x^{2}=1.413$ & 0.493 \\
Postoperative hospital stay (days) & Minimally Invasive & $4(5-4)$ & $5(5.5-4)$ & $5(6-4)$ & $x^{2}=5.360$ & 0.069 \\
& Open & $4.5(6.5-3.5) *$ & $6(7-5)$ & $5(6-4) *$ & $X^{2}=12.248$ & 0.002 \\
\hline
\end{tabular}

Operation time, blood loss, and postoperative hospital stay are expressed as median (75\% quartile-25\% quartile). $R M D$ rank means difference

*Represents statistical significance compared with the spondylolisthesis group $(p<0.05)$, and $\Delta$ represents statistical significance compared with disc herniation group $(p<0.05)$ undergoing same surgical method (minimally invasive group or open group)

root. Most foot drop cases after lumbar internal fixation are related to this issue $[4,9]$. The incidence of nerve root irritation during pedicle screw insertion can be as high as $21 \%$, of which a considerable number of patients have poorly positioned screws but are asymptomatic [2]. In addition, screws of inappropriate length may penetrate the vertebra, injuring blood vessels or adjacent organs. With the development of robot-assisted screw placement technology, the length and angle of screw placement are finely controlled. The accuracy and safety of screw placement have been further improved, and iatrogenic injuries have gradually decreased. Compared with traditional freehand screw insertion, the acceptability of robot-assisted screw insertion can reach 95-99\% [11, 12], which matches the $97.3 \%$ acceptability rate in our RAMIS group and $95.6 \%$ in the RA-OS group.

Soft tissues connect bony structures, ligaments, discs, and muscles, and are important structures in maintaining spinal stability. Damage to the spinal accessory tissues reduces surgical efficacy and increases the risk of complications. The incidence of surgical infections after instrument surgery is relatively high, which may be related to the greater soft tissue dissection, large surgical wounds, and use of instruments [17, 22]. Minimally invasive spine 
surgery decreases intraoperative blood loss, shortens postoperative hospital stay, and reduces tissue damage. Robot-assisted minimally invasive spine surgery is a new development direction in spinal surgery $[14,20]$.

In this study, there was no statistical difference in screw deviation, Gertzbein and Robbins grade distribution, and facet joint invasion between the RA-MIS and RA-OS groups. Screw deviation in the RA-MIS group was smaller than that in the RA-OS group but with no statistical difference, which was better than the $2.0 \pm 1.2$ deviation reported by van Dijk et al. using the Mazor robot [19]. Regarding small joint invasion, the $2-3 \%$ invasion rate in our study was better than the $7 \%$ invasion rate in the robot group reported by Archavlis et al. [1]. This showed that the positioning accuracy and safety of the TiRobot has reached the international robotic level. While preserving more tissues around the spine, RA-MIS can maintain the same accuracy and safety as RA-OS.

Regarding the perioperative outcomes in this study, the RA-MIS group had statistically significantly less intraoperative blood loss and shorter postoperative hospital stay than the RA-OS group. Less blood loss may be related to the smaller surgical incision, less muscle dissection, and less intraoperative vascular damage, intraoperatively. The shorter postoperative hospital stay may have resulted from less postoperative bleeding and drainage [13, 15]. Compared with RA-OS, RA-MIS had statistically significant effects in reducing intraoperative blood loss. Additionally, one patient in the RA-MIS group underwent postoperative expansion surgery compared with none in the RA-OS group. This may be related to wound infection caused by the patient's second-degree obesity $(\mathrm{BMI}=31.25)[5]$.

We also performed a subgroup analysis by disease and compared the RA-MIS and RA-OS groups. For RA-MIS, screw deviation in the fracture subgroup was smaller than that in the spondylolisthesis subgroup and the disc herniation subgroup. The $99.18 \%$ screw placement acceptance rate in the fracture subgroup was better than the $97.7 \%$ acceptance rate described by Lin et al. [13], and the rate in our spondylolisthesis subgroup was better than data reported by Cui et al. ( $95.8 \%$ vs $93.8 \%$, respectively) [3, 13]. The difference between the subgroups may be related to the degree of damage to the vertebral body. In particular, for patients with spondylolisthesis, the screw precision in the RA-MIS group was higher $(p=0.036)$, and the postoperative hospital stay was shorter $(p<0.001)$ compared with RA-OS. This may be related to the better preservation of intervertebral joint structures and less muscle damage in patients undergoing MIS. Regarding the perioperative outcomes, intraoperative blood loss was lower and operation time was shorter in the fracture subgroup than in the other groups. This may be because of easier fracture treatment and the relatively short postoperative immobilization time.

In summary, our results indicated that RA-MIS and RA-OS have equal accuracy and safety. MIS was associated with less intraoperative blood loss in each subgroup and a shorter postoperative hospital stay in the spondylolisthesis subgroup. Our subgroup analysis of MIS showed that the fracture group experienced less blood loss, shorter operation time, and shorter postoperative hospital stay compared with the spondylolisthesis group and disc herniation group. This study has the following shortcomings: there were few relevant published references; therefore, comparisons with others studies were not comprehensive. Additionally, we did not evaluate bone density, which may have caused confounding bias.

\section{Conclusions}

RA-OS and RA-MIS had the same accuracy and safety. Compared with open surgery, minimally invasive surgery reduced blood loss in all fracture subgroup, spondylolisthesis subgroup as well as disc herniation subgroup, and shortened the postoperative hospital stay in the spondylolisthesis subgroup. Compared with the other subgroups under minimally invasive surgery, the fracture subgroup had less blood loss and shorter operation time. This paper studied the effect of robotic-assisted minimally invasive surgery screws in different diseases, and provided a basis for economic benefit research.

\section{Abbreviations}

RA-MIS: Robot-assisted minimally invasive surgery; RA-OS: Robot-assisted open surgery; BMI: Body mass index; CT: Computed tomography; ANOVA: Analysis of variance; RMD: Rank mean difference.

\section{Acknowledgements}

Not applicable.

Authors' contributions

FMX and FYM contributed equally to this article. FMX and FYM together designed the work and analysed the data. ZQ and ZJW collected the data acquisition. LB and TW participated in designing the work, while TW was the corresponding author of this paper. All authors are accountable for the contributions. All authors read and approved the final manuscript.

\section{Funding}

Natural Science Foundation of Beijing Municipality (L212062), Beijing Outstanding Talent Training Foundation (CN) (2018000021469G225); Beijing Jishuitan Hospital youth talent training project (XKXX202104). The funder plays no roles in our study.

Availability of data and materials

The datasets used and/or analysed during the current study are available from the corresponding author on reasonable request.

\section{Declarations}

Ethics approval and consent to participate

This study was approved by the Ethics Committee of Beijing Jishuitan Hospital (No. 202007-14). Written informed consent was collected form patients. The 
study was performed in accordance with the ethical standards in the 1964 Declaration of Helsinki and its later amendments or comparable ethical standards.

\section{Consent for publication}

Images are entirely unidentifiable and there are no details on individuals reported within the manuscript.

\section{Competing interests}

The authors declare that they have no competing interests.

\section{Author details}

${ }^{1}$ Spine Department, Beijing Jishuitan Hospital, Beijing, China. ${ }^{2}$ Beijing Key Laboratory of Robotic Orthopaedics, Beijing, China.

Received: 28 October 2021 Accepted: 31 January 2022

Published online: 11 February 2022

\section{References}

1. Archavlis E, Amr N, Kantelhardt SR, et al. Rates of upper facet joint violation in minimally invasive percutaneous and open instrumentation: a comparative cohort study of different insertion techniques. J Neurol Surg A Cent Eur Neurosurg. 2018;79(1):1-8. https://doi.org/10.1055/s-00371603631.

2. Bose B, Wierzbowski LR, Sestokas AK. Neurophysiologic monitoring of spinal nerve root function during instrumented posterior lumbar spine surgery. Spine (Phila Pa 1976). 2002;27(13):1444-50. https://doi.org/10. 1097/00007632-200207010-00014.

3. Cui GY, Tian W, He D, et al. Effects of robot-assisted minimally invasive transforaminal lumbar interbody fusion and traditional open surgery in the treatment of lumbar spondylolisthesis. Zhonghua Wai Ke Za Zhi. 2017;55(7):543-8. https://doi.org/10.3760/cma.j.issn.0529-5815.2017.07. 013.

4. El Mansy Y, Migliorini F, Tingart M, et al. Minimally versus conventionalinvasive transforaminal lumbar interbody fusion in patients with failed back surgery syndrome. Musculoskelet Surg. 2020. https://doi.org/10. 1007/s12306-020-00659-7.

5. Elgafy $\mathrm{H}, \mathrm{Hamilton} \mathrm{R}$, Peters $\mathrm{N}$, et al. Critical care of obese patients during and after spine surgery. World J Crit Care Med. 2016;5(1):83-8. https://doi. org/10.5492/wjccm.v5.i1.83.

6. Gelalis ID, Paschos NK, Pakos EE, et al. Accuracy of pedicle screw placement: a systematic review of prospective in vivo studies comparing free hand, fluoroscopy guidance and navigation techniques. Eur Spine $J$. 2012;21(2):247-55. https://doi.org/10.1007/s00586-011-2011-3.

7. Gertzbein SD, Robbins SE. Accuracy of pedicular screw placement in vivo. Spine (Phila Pa 1976). 1990;15(1):11-4. https://doi.org/10.1097/00007632199001000-00004.

8. Han X, Tian W, Liu Y, et al. Safety and accuracy of robot-assisted versus fluoroscopy-assisted pedicle screw insertion in thoracolumbar spinal surgery: a prospective randomized controlled trial. J Neurosurg Spine. 2019. https://doi.org/10.3171/2018.10.Spine18487.

9. Harimaya K, Lenke LG, Son-Hing JP, et al. Safety and accuracy of pedicle screws and constructs placed in infantile and juvenile patients. Spine (Phila Pa 1976). 2011;36(20):1645-51. https://doi.org/10.1097/BRS.0b013 e318225b8f9.

10. Hayashi D, Roemer FW, Mian A, et al. Imaging features of postoperative complications after spinal surgery and instrumentation. AJR Am J Roentgenol. 2012;199(1):W123-129. https://doi.org/10.2214/ajr.11.6497.

11. Le X, Tian W, Shi Z, et al. Robot-assisted versus fluoroscopy-assisted cortical bone trajectory screw instrumentation in lumbar spinal surgery: a matched-cohort comparison. World Neurosurg. 2018;120:e745-51. https://doi.org/10.1016/j.wneu.2018.08.157.

12. Lieberman $1 \mathrm{H}$, Kisinde $\mathrm{S}$, Hesselbacher S. Robotic-assisted pedicle screw placement during spine surgery. JBJS Essent Surg Tech. 2020;10(2): e0020. https://doi.org/10.2106/jbjs.St.19.00020.

13. Lin S, Hu J, Wan L, et al. Short-term effectiveness comparison between robotic-guided percutaneous minimally invasive pedicle screw internal fixation and traditional open internal fixation in treatment of thoracolumbar fractures. Zhongguo Xiu Fu Chong Jian Wai Ke Za Zhi. 2020;34(1):76-82. https://doi.org/10.7507/1002-1892.201906105.

14. Minamide A, Yoshida M, Iwahashi $\mathrm{H}$, et al. Minimally invasive decompression surgery for lumbar spinal stenosis with degenerative scoliosis: predictive factors of radiographic and clinical outcomes. J Orthop Sci. 2017;22(3):377-83. https://doi.org/10.1016/j.jos.2016.12.022.

15. Momin AA, Steinmetz MP. Evolution of minimally invasive lumbar spine surgery. World Neurosurg. 2020;140:622-6. https://doi.org/10.1016/j. wneu.2020.05.071.

16. Nimjee SM, Karikari IO, Carolyn AHAB, et al. Safe and accurate placement of thoracic and thoracolumbar percutaneous pedicle screws without image-navigation. Asian J Neurosurg. 2015;10(4):272-5. https://doi.org/ 10.4103/1793-5482.162700.

17. Ojo OA, Owolabi BS, Oseni AW, et al. Surgical site infection in posterior spine surgery. Niger J Clin Pract. 2016;19(6):821-6. https://doi.org/10. 4103/1119-3077.183237.

18. Swann MC, Hoes KS, Aoun SG, et al. Postoperative complications of spine surgery. Best Pract Res Clin Anaesthesiol. 2016;30(1):103-20. https://doi. org/10.1016/j.bpa.2016.01.002.

19. van Dijk JD, van den Ende RP, Stramigioli S, et al. Clinical pedicle screw accuracy and deviation from planning in robot-guided spine surgery: robot-guided pedicle screw accuracy. Spine (Phila Pa 1976). 2015;40(17):E986-991. https://doi.org/10.1097/brs.0000000000000960.

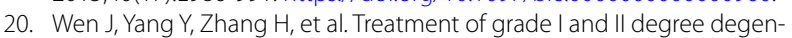
erative lumbar spondylolisthesis with minimally invasive surgery-transforaminal lumbar interbody fusion under Quadrant channel. Zhongguo Gu Shang. 2019;32(3):199-206. https://doi.org/10.3969/j.issn.1003-0034.2019. 03.002.

21. Zhang JN, Fan Y, He X, et al. Comparison of robot-assisted and freehand pedicle screw placement for lumbar revision surgery. Int Orthop. 2020. https://doi.org/10.1007/s00264-020-04825-1.

22. Zhou J, Wang R, Huo $X$, et al. Incidence of surgical site infection after spine surgery: a systematic review and meta-analysis. Spine (Phila Pa 1976). 2020;45(3):208-16. https://doi.org/10.1097/brs.0000000000003218.

\section{Publisher's Note}

Springer Nature remains neutral with regard to jurisdictional claims in published maps and institutional affiliations.

Ready to submit your research? Choose BMC and benefit from

- fast, convenient online submission

- thorough peer review by experienced researchers in your field

- rapid publication on acceptance

- support for research data, including large and complex data types

- gold Open Access which fosters wider collaboration and increased citations

- maximum visibility for your research: over $100 \mathrm{M}$ website views per year

At BMC, research is always in progress.

Learn more biomedcentral.com/submissions 